\title{
The return of the phage
}

\author{
Technological advances enable scientists to engineer and use phages to treat a wide range of diseases \\ including cancer
}

Philip Hunter

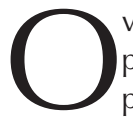
ver the past decade, bacteriophages have occasionally stirred public and media interest because of their potential as biological weapons against bacterial infections. Such reports have tended to come from Russian or Georgian laboratories, whereas Western research institutes and companies have usually found that phages do not live up to their promise. More than a decade later, however, the view of bacteriophages is set to change. Spurred on by advances in sequencing and other molecular techniques, research into phages has yielded its first applications. Not only are phages proving effective as therapeutic agents, but they are also playing a role in food safety and as delivery vehicles for drugs against a wide range of diseases.

\section{...it is only in the past five years that the regulatory guidelines for the approval of phage products - both in therapy and food safety-have been created}

Interest in phages as therapeutic agents emerged almost immediately after their discovery nearly a century ago (Twort, 1915; d'Hérelle, 1917). This interest evaporated quickly in the West after the discovery of penicillin, but phage research was kept alive in the old Soviet Union and continued after its collapse in the 1990s. Ongoing studies there, although not always conforming to the most rigorous standards, provided the only evidence of the therapeutic potential of phages.

Eventually, especially in the light of the increasing threat from drug-resistant bacteria, Western researchers turned to exploring phages again. However, it is only in the past five years that the regulatory guidelines for the approval of phage products -in both therapy and food safety-have been created. Previously, the US Food and Drug Administration (FDA) had lacked the appropriate regulatory measures; it took them four years to approve the first phage product for use in food safety in 2006. ListShield ${ }^{\mathrm{TM}}$ is a cocktail of several phages that target Listeria monocytogenes, contaminants in meat and poultry products. Approvals for other food safety products have followed with greater speed (Sulakvelidze, 2011). Moreover, in 2008, the FDA approved the first phase 1 clinical trial of phages. This again involved a cocktail of eight phages to target various bacteria including Staphylococcus aureus, Pseudomonas aeruginosa and Escherichia coli, in venous leg ulcers. This trial eventually established the safety of the phage preparation and cleared the way for more phage therapy trials (www. clinicaltrials.gov).

$\mathrm{T}$ he recent acceptance in the West of phages as anti-pathogenic agents was preceded by their use for diagnostic purposes to identify bacteria, according to Martin Loessner from the Institute of Food, Nutrition and Health in Zürich, Switzerland. "It then became possible to [...] harness the specificity of phage for applications such as recognition of the host cell, and also for reporter phage, which is a genetically modified phage with a gene so [you] can easily see the phage's impact on the target cell," he explained. "Later on we figured why not go and revisit the idea of using phages against pathogens."
This approach turned out to be highly successful against key food pathogens, Loessner said, because of the way phages work: "[T] he phage has been very finely tuned through zillions of generations in the evolutionary arms race, and is highly specific." This specificity is important for targeting the few bacteria that cause food poisoning while sparing the bacteria in fermented food-such as soft cheeses - that are harmless and contribute flavour. "The phage is also immune to development of resistance by the host bacteria, because if not it would have become extinct a long time ago," Loessner said.

\section{The recent acceptance in the West of phages as anti- pathogenic agents was preceded by their use for diagnostic purposes to identify bacteria...}

It is bacterial toxins that cause food poisoning rather than bacteria themselves, so phages are used as a preventive measure to stop the growth of bacteria such as Listeria in the first place. As such, it is important to bombard food products with a large number of phages to ensure that virtually all target bacteria are eradicated. "I always have this magic number of $10^{8}$, or 100 million per gram of food," Loessner said. "In $1 \mathrm{~g}$ of food there are often only 500 target bacteria, so there is not enough to amplify the phage and you need really high numbers to kill the bacteria in one round of infection." He added that, in his view, phages would soon become the main treatment for preventing bacterial contamination. "Phage in the near future will be 
the number one [treatment against] Listeria and Salmonella. It's becoming number one already, especially in the US."

In Europe, the use of phages in food safety therapy is being held back by the requirement that foods treated with them are labelled as containing viruses, which means they are likely to meet consumer resistance, as happened with foods containing or made from genetically modified organisms. Loessner commented that education is required to raise awareness that the properly controlled use of phages involves minimal risk and could greatly enhance food safety. However, he also emphasized that the use of phages should represent an extra level of protection, not replace existing quality control measures.

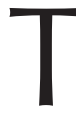

he ability of phages to target specific bacteria while leaving others alone also has great potential for treating bacterial infections, particularly in the light of increasing antibiotic resistance. Such treatments would not necessarily involve the phage themselves, but rather the use of their lysins-the enzymes that weaken the bacterial cell wall to allow newly formed viruses to exit the host cell. Lysins can be administered as antibiotics, at least for gram-positive bacteria that lack a separate outer membrane around the cell wall. Moreover, because phage lysins are often specific to a single bacterial genus, they would allow the specific targeting of pathogenic bacteria. "The fact that phage lysins leave the commensal microflora undisturbed is particularly significant," commented Olivia McAuliffe, Senior Research Officer at the Teagasc Food Research Centre in Cork, Ireland. "Most of the antibiotics used clinically have broadactivity spectra and treatment with these antibiotics can have devastating effects on the normal flora, in particular for those taking long-term antibiotic courses."

Phages also have another great advantage over most conventional antibiotics in being potent against both dividing and non-dividing cells. "Because most antibiotics target pathways such as protein synthesis, DNA replication, and cell wall biosynthesis, they can only act when the

\section{...because phage lysins are often} specific to a single bacterial genus, they would allow the specific targeting of pathogenic bacteria

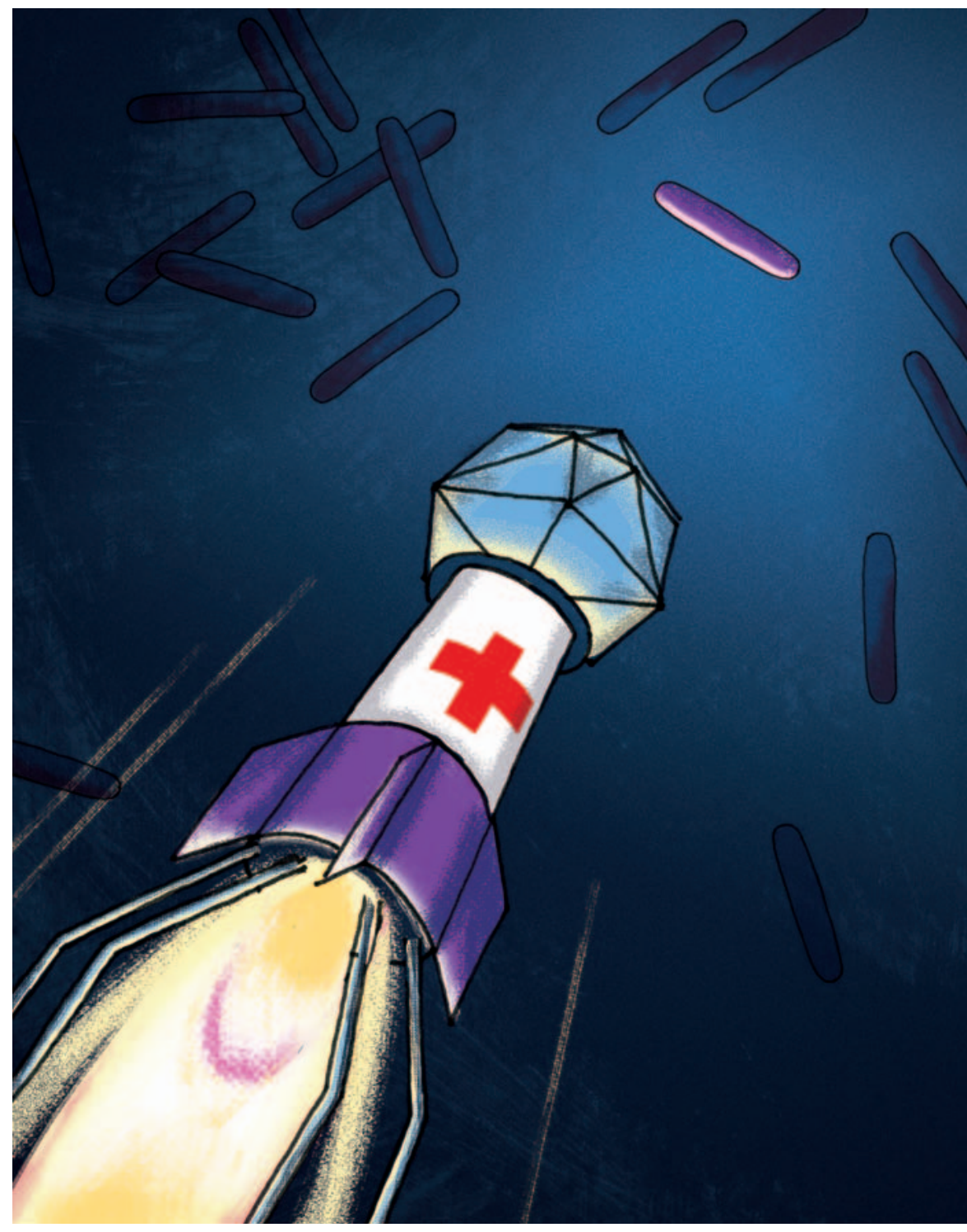

cells are actively growing," McAuliffe added. "Because lysins are enzymes, they will chew away the peptidoglycan in both viable and non-viable cells, dividing and non-dividing cells. This would be particularly important in the case of slow-growing organisms that cause infection, an example being Mycobacterium species."

This specificity of phages and their lysins is particularly important for treating chronic conditions resulting from persistent bacterial infection, particularly in the respiratory system or digestive tract. Broadspectrum antibiotics also attack harmless and beneficial commensal bacteria, and can even worsen the condition by encouraging the growth of resistant bacteria. This is the case with Clostridium difficile, a cause of secondary infections and a major nosocomial (hospital-acquired) antibioticresistant pathogen, according to McAuliffe. It is a Gram-positive, rod-shaped, sporeforming bacterium that is the most serious and common cause of diarrhoea and other intestinal disease when competing bacteria in the gut flora have been wiped out by antibiotics. The bacterium and its spores, which form in aerobic conditions outside the body, are widespread in the environment and are present in the guts of $3 \%$ of healthy individuals and $66 \%$ of infants, according to the UK's Health Protection Agency. Clostridium spreads readily on the hands of healthcare staff and visitors in hospitals. The ability of the bacteria to form spores resistant to heat, drying and disinfectants, which then adhere to surfaces, enables them to persist in the hospital environment. 
Because Clostridium is resistant to most conventional antibiotics, it has for some years usually been treated with metronidazole, which exploits the fact that Clostridium is anaerobic during infection. Metronidazole has proven particularly appealing as it has relatively little impact on human cells or commensal aerobic bacteria in the gut as it does not work in the presence of oxygen. But metronidazole does not always work, and physicians have therefore been using vancomycin, a stronger but more toxic antibiotic, as a last resort. Moreover, even in cases where antibiotics seem to eliminate Clostridium and cure the associated diarrhoea, infection recurs in as many as $20 \%$ of hospital patients (Kelly \& LaMont, 2008). About one-fifth of these $20 \%$, or $4 \%$ of the total number of patients succumbing to Clostridium, end up with a long-term infection that at present is difficult to eradicate.

\section{Modified phages could also serve as nanoparticles to deliver cytotoxic drugs straight to tumour cells, bypassing healthy cells}

This is where phages step in, because they are well tolerated by patients and their specificity means that they will not target other gut bacteria. Clostridium phages have already been demonstrated to work selectively and there is the possibility of extracting lysins against Clostridium from the phage itself; an avenue being pursued by Aidan Coffey's group at the Department of Biological Sciences at the Cork Institute of Technology in Bishoptown, Ireland.

There is also growing interest in using phages to tackle various other infections that are resistant to existing drugs-for example, in wounds that fail to heal, which are a major risk for diabetics. The application of phages in such cases is not new-before penicillin it was often the only option-but the difference now is that modern molecular techniques for isolating bacterial strains from biopsies and matching them to phages greatly increases efficiency. One clinical trial, organized by the Institute of Immunology and Experimental Therapy of the Polish Academy of Sciences, is currently recruiting patients to evaluate the use of phage preparations against a range of drug-resistant bacteria, including MRSA (methicillin-resistant Staphylococcus aureus), Enterococcus,
Escherichia, Citrobacter, Enterobacter, Klebsiella, Shigella and Salmonella. The intention is to isolate bacterial strains from each patient and to identify matching phages from the Institute's bacteriophage collection in Wrocław.

A Ithough the potential of phages or their lysins to combat bacterial pathogens, whether in food or those causing infectious diseases, has long been recognized, more recent work has identified new applications as delivery vehicles for vaccines or cytotoxic drugs to treat cancer. These applications do not exploit the phage's natural targeting of bacteria, but make use of their ability to carry surface ligands that attract them to specific host cells.

Even though phages do not attack human cells, they elicit an immune response and can be used as vectors to carry an engineered antigen on their surface to vaccinate against viral or bacterial disease. This approach has been tested in rabbits with a DNA vaccine against hepatitis B (Clark et al, 2011). The study compared the phage DNA vaccine with Engerix B-a commercially available vaccine based on a homologous recombinant protein-and found that the phage vaccine produced a significantly higher antibody response more quickly, as well as being potentially cheaper to produce and stable at a wider range of temperatures. This hepatitis $B$ vaccine is now being developed by the UK biotech firm BigDNA in Edinburgh, Scotland, which has been granted a European patent, pending future clinical trials in humans.

Modified phages could also serve as nanoparticles to deliver cytotoxic drugs straight to tumour cells, bypassing healthy cells. Phages are a promising candidate vehicle because they can be readily engineered both to display appropriate ligands for targeting tumour cells specifically, and to carry a cytotoxic payload that is only released inside the target. One Israeli group has developed a technology for manufacturing phage nanoparticles that in principle can be used to target drugs to either tumour cells or pathogens (Bar et al, 2008). The group chose one particular phage family, known as filamentous phages, because of their small size and the relative ease of engineering them. Filamentous phages comprise just 10 genes with a sheath of several thousand identical a-helical coat proteins in a helical array assembled around a single-stranded circular DNA molecule. The Israeli scientists combine genetic modification and chemical engineering to create a phage that is able to attach to its target cell and release cytotoxic molecules. "Genetic engineering makes it possible to convert the phage to a targeted particle by displaying a target-specifying molecule on the phage coat," explained Itai Benhar from Tel-Aviv University, the lead author of the paper. "Genetic engineering also makes it possible to design a drug-release mechanism. Finally chemical engineering makes it possible to load the particle with a large payload of cargo."

\section{The use of phages is no longer confined to directly attacking infectious bacteria, but has vastly expanded in terms of methods, applications and the diseases that can be tackled}

The group has used the same approach to target two bacteria species, Staphylococcus aureus and Escherichia coli, with the antibiotic chloramphenicol, which was first developed in 1949 but has raised concerns over its toxicity. According to the Israeli group, the phage nanoparticle loaded with the drug was 20,000 times more potent against both bacteria than the drug administered on its own. Just as importantly, the phage particles do not affect other cells. The overall advantage of the phage-based delivery approach is that it can deliver highly effective and toxic drugs in a safe way. The other point is that this and other methods in which phages are engineered to reach specific targets have nothing directly to do with the natural ability of phage viruses to attack bacteria. "The phage's natural ability to infect bacteria is totally irrelevant to their application for targeting non-bacterial cells," said Benhar. "In fact, they are not relevant for targeting bacteria either in this case, since the chemical modification we subject the phages to renders them non-infective."

However, the phage nanoparticles retain their immunogenic effect, which is a problem if the objective is merely to deliver a drug to the target while minimizing all other impacts. "Phages are immunogenic, and although we found a way to reduce their 
immunogenicity we did not totally eliminate it," Benhar said. The other challenge is that, as the particles carry the payload drug on their surface, the physical and chemical properties change every time a new drug is loaded. Although the payload itself is inert until it reaches the target, the varying characteristics could alter the host response and therefore affect regulatory approval for each new phage construct, as safety would have to be demonstarted in each case.

Nevertheless, this approach holds great promise as a novel way of delivering not just new drugs but also existing ones that are effective but too toxic for healthy cells. This is exactly the most exciting aspect of recent therapeutic phage research. The use of phages is no longer confined to directly attacking infectious bacteria, but has vastly expanded in terms of methods, applications and the diseases that can be tackled.

\section{CONFLICT OF INTEREST}

The author declares that he has no conflict of interest.

\section{REFERENCES}

Bar H, Yacoby I, Benhar I (2008) Killing cancer cells by targeted drug-carrying phage nanomedicines. BMC Biotechno/ 8: 37

Clark JR, Bartley K, Jepson CD, Craik V, March JB (2011) Comparison of a bacteriophage-delivered DNA vaccine and a commercially available recombinant protein vaccine against hepatitis $B$. FEMS Immunol Med Microbiol 61: 197-204
d'Hérelle F (1917) Sur un microbe invisible antagoniste des bacilles dysentériques. CR Acad Sci Ser D 165: 373-375

Kelly CP, LaMont JT (2008) Clostridium difficilemore difficult than ever. N Engl / Med 359: 1932-1940

Sulakvelidze A (2011) Safety by nature: potential bacteriophage applications. Microbe 6: 122-126

Twort FW (1915) An investigation on the nature of the ultramicroscopic viruses. Lancet 186: 1241-1243

Philip Hunter is a freelance journalist in London, UK.

EMBO reports (2012) 13, 20-23; published online 2 December 2011; doi:10.1038/embor.2011.234 